\title{
Highly sensitive measurements of radioactive noble gas nuclides in the BOREXINO solar neutrino experiment
}

\author{
H. Simgen", G. Heusser, G. Zuzel \\ Max-Planck-Institut für Kernphysik, P.O. Box 103980, D-69029 Heidelberg, Germany \\ * Corresponding author. Tel: $\quad+49-6221-516-530$ \\ Fax: +49-6221-516-324 \\ Email: Hardy.Simgen@mpi-hd.mpg.de
}

Keywords: BOREXINO, chromatography, noble gases, proportional counter, solar neutrinos

\begin{abstract}
Low background miniaturized proportional counters as developed for the GALLEX solar neutrino experiment can be applied to the detection of radioactive noble gas nuclides at very low activities. We have developed an apparatus that allows the activity of trace amounts of isotopes of the four noble gases $\mathrm{Ar}, \mathrm{Kr}, \mathrm{Xe}$ and $\mathrm{Rn}$ to be measured. The technique includes contamination-free chromatographic purification of raw gas samples and subsequent lowlevel counting. Minimum detectable activities of $100 \mu \mathrm{Bq}$ and below have been attained. The developed techniques can be used to determine the ${ }^{222} \mathrm{Rn}$ and ${ }^{85} \mathrm{Kr}$ concentration in nitrogen for the solar neutrino experiment BOREXINO. By applying efficient techniques to concentrate noble gases from nitrogen, minimum detectable activity concentrations below $1 \mu \mathrm{Bq} / \mathrm{m}^{3}$ of nitrogen (STP) have been reached for both nuclides.
\end{abstract}

\section{Introduction}

Radioactive noble gas nuclides are an important source of background in experiments aiming to detect events with very low count-rates. In this paper we present a way to measure the activity of radioisotopes of the four noble gases $\mathrm{Ar}, \mathrm{Kr}, \mathrm{Xe}$ and $\mathrm{Rn}$ at very low concentrations with proportional counters. The counters were originally developed for the GALLEX solar neutrino experiment (Wink et al., 1993) and were later used in the BOREXINO project for ${ }^{222} \mathrm{Rn}$ measurements (Heusser et al., 2000; Rau and Heusser, 2000; Simgen et al., 2003a). The work was motivated by the need for a sensitive measurement of ${ }^{85} \mathrm{Kr}$ in the BOREXINO experiment. However, we did not only focus on this application but built a multi-purpose instrument that can be used for all noble gases heavier than neon. The key issue for a low detection limit is an efficient purification of the gas sample before it is filled into the counter. Consequently we developed and tested purification procedures for each of the four noble gases. 


\section{Purification of noble gas samples}

\subsection{Purification line}

The purification line (see Figure 1) for the gas sample is a glass vacuum apparatus similar to the unit used in the GNO experiment (Richter, 1988). Its main component is a gas chromatography column for the separation of the different gas components of a sample. Its length is about $75 \mathrm{~cm}$ and its internal diameter is $0.6 \mathrm{~cm}$. The gases are detected at the end of the column in a thermistor block that measures the heat conductivity of the passing gases. The adsorber in the column can be exchanged. Presently $6 \mathrm{~g}$ of Chromosorb $102^{\mathrm{TM}}$ are used for all applications.

\subsection{The purification procedure}

Many different tests were performed to optimize the operation parameters of the apparatus (Simgen, 2003b). Finally procedures were developed that allow a good separation for each of the noble gases heavier than neon from all other components $\left(\mathrm{N}_{2}, \mathrm{O}_{2}, \mathrm{CO}_{2}, \ldots\right)$. It has also been checked that the gas of interest is completely transferred through the line during different operations. Merging the result from all the test series, the final procedure is as follows:

A vial containing the gas sample to be purified is mounted to the line. The sample is extracted from the vial with helium carrier gas (flow rate: $\sim 3$ liters/h). $\mathrm{CO}_{2}$ can be removed in a trap with moist $\mathrm{NaOH}$ and the sample can be dried in a silica gel trap. Then, the gasses are flowed over the chromatography column that is cooled to $77 \mathrm{~K}$. The heavy noble gases and most of the other impurities including nitrogen are frozen out at the top of the column. When the column is warmed up, the gases move through it with a velocity dependent on their adsorption coefficient. Chromosorb 102 ${ }^{\mathrm{TM}}$ is an adsorber with distinct pores (about $8.5 \mathrm{~nm}$ ). The absence of micropores $(<2 \mathrm{~nm})$ in which gas particles would be adsorbed strongly leads to symmetric narrow peaks in the chromatograms and consequently to an efficient separation. The following part of the purification procedure varies for different gases and will be explained for each of them individually.

\subsubsection{Gas chromatography with noble gases}

\section{Argon}

Argon is difficult to separate from nitrogen (and oxygen) because these gases have similar adsorption properties. This can be seen by comparing their adsorption parameters, defined as the ratio of the critical pressure and the square root of the critical temperature (Maurer et al., 2001). Low temperatures and consequently long retention times provide the best possibility to separate these gases. Therefore we operate the column at $165 \mathrm{~K}$. Although a complete separation is not possible, the amount of nitrogen can be reduced down to a level that can be adsorbed by a getter-pump (see section 2.2.2). After the separation the argon is trapped in a $0.6 \mathrm{~g}$ activated carbon trap at $77 \mathrm{~K}$. Activated carbon was chosen because its microporosity in combination with a low temperature offers the most effective retention ability for argon. 


\section{Krypton}

If krypton has to be measured, the column is warmed up to $215 \mathrm{~K}$. This temperature was chosen because it still allows a good separation of krypton, while on the other hand maintaining a high velocity compared to lower temperatures. Thus the separation process is substantially accelerated. For the recovery of krypton one trap with Chromosorb $102^{\mathrm{TM}}$ and two activated carbon traps are available (see Figure 1). Usually the Chromosorb $102^{\mathrm{TM}}$ trap is preferred, because the krypton can be easily desorbed in the subsequent step. If methane is present in the sample, the separation by chromatography becomes difficult, because the adsorption parameters and therefore the velocities are almost identical. In this case it might be necessary to remove methane with a getter-pump.

\section{Xenon}

In the case of xenon the column is operated at $250 \mathrm{~K}$. The same traps as in the case of krypton are utilized and again the Chromosorb $102^{\mathrm{TM}}$ trap is the preferred one. Another feature has to be mentioned: $\mathrm{CO}_{2}$ has a slightly smaller adsorption parameter than xenon resulting in a somewhat shorter retention time. Therefore $\mathrm{CO}_{2}$ can be used as an indicator for the appearance of xenon. This is helpful because often the xenon peak in the chromatogram is not visible since the amount to be measured is very small (sometimes only few atoms). Because the operation parameters are not completely identical in each run, the retention times vary slightly. If an indicator like $\mathrm{CO}_{2}$ is available, a control of the performance of the column during the run becomes possible.

\section{Radon}

Techniques to purify and measure ${ }^{222} \mathrm{Rn}$ with proportional counters have been developed earlier in BOREXINO. They are described in detail in (Heusser et al., 2000; Rau and Heusser, 2000, Simgen et al., 2003a). The present system described in this work is also able to handle radon samples. A chromatography run is usually not necessary to separate the radon. The whole sample is transferred to a Chromosorb $102^{\mathrm{TM}}$ trap at $77 \mathrm{~K}$. After warming up the trap to $165 \mathrm{~K}$ most of the gaseous contaminations can be pumped away while radon remains in the trap.

For each of the noble gases the column is operated at a different temperature. If the temperature is increased step by step, it is possible to separate more than one of the noble gases from the same sample. Because three traps are available for collecting the gases released from the chromatography column, up to three noble gases can be extracted from one sample. This is important for samples that rare or difficult to obtain.

\subsubsection{Final purification and counter filling}

After the separation process the noble gases are stored in one of the three traps following the chromatography column. The sample is still mixed with the carrier gas helium. In the case of radon and xenon the helium can be pumped away at $165 \mathrm{~K}$. For krypton and argon the trap can not be warmed up and is held at $77 \mathrm{~K}$ during pumping. Helium can not be removed completely at this temperature, but the residual amount is sufficiently small to not disturb the counting (about $0.1 \mathrm{~cm}^{3}$ of helium to be compared with the volume of the counter: $\sim 1 \mathrm{~cm}^{3}$ ). Traces of other gases which at this stage are still present are removed by a zirconium/aluminium getter-pump. 
After this final purification a Toepler-pump (for a description see e.g. Shriver, 1969) is used to mix the sample with the counting gas and insert it into the counter. For the counting gas different choices are possible. Usually the gas consists of $10 \%$ quencher (methane) and $90 \%$ noble gas. The best energy resolution is obtained with argon. Xenon on the other hand has the advantage that due to its higher atomic number radiation deposits more energy in the small counter volume resulting in a higher efficiency. Moreover xenon offers the possibility for an energy calibration at low energies by using a source that excites xenon x-rays. The same technique is used in the GNO experiment and is described in (Schanda and Urban, 1996). Another reason why xenon is preferred is because xenon has no long-lived isotopes.

\section{Low background counting}

To reduce the cosmogenic induced background the counting setup is located in an underground laboratory in Heidelberg (15 m w.e.). The counter is placed inside a multiwell plastic scintillator block that provides a veto for cosmic muons. The scintillator is surrounded by about $15 \mathrm{~cm}$ of lead. The integral background rate above an energy threshold of $600 \mathrm{eV}$ depends on the individual counter and lies in the range of several events per day The counting efficiency depends on the decay mode of the investigated isotope. We focused on the nuclides ${ }^{39} \mathrm{Ar},{ }^{85} \mathrm{Kr},{ }^{222} \mathrm{Rn}$ and ${ }^{133} \mathrm{Xe}$. Our interest in the first three of these nuclides is motivated by the fact that they have the highest concentration in the atmosphere (see Table 1) and therefore they are serious background sources for the BOREXINO experiment. The ${ }^{235} \mathrm{U}$ fission product ${ }^{133} \mathrm{Xe}$ was investigated because it offers the possibility for a sensitive measurement of uranium.

${ }^{39} \mathrm{Ar},{ }^{85} \mathrm{Kr}$ and ${ }^{133} \mathrm{Xe}$ are detected in proportional counters by their $\beta$-decays, while ${ }^{222} \mathrm{Rn}$ is detected by its $\alpha$-decay. $\beta$-particles of several hundred keV do not deposit much energy in the active volume of the counter. Therefore we apply the lowest possible energy threshold of $600 \mathrm{eV}$ in order to get a high counting efficiency (for lower thresholds the background increases strongly). On the other hand $\alpha$-particles deposit much more energy in the counter so that a threshold of $50 \mathrm{keV}$ can be applied. The background is reduced in that case to less than 0.5 events per day

The efficiency calibration was performed with ${ }^{85} \mathrm{Kr}$ and ${ }^{222} \mathrm{Rn}$ activity standards. The efficiencies for ${ }^{39} \mathrm{Ar}$ and ${ }^{133} \mathrm{Xe}$ detections should not differ significantly from the result obtained for ${ }^{85} \mathrm{Kr}$ due to the similar energy distribution of their $\beta$-particles. We measured a total counting efficiency of $(48 \pm 3) \%$ for ${ }^{85} \mathrm{Kr}$ when applying an energy threshold of $600 \mathrm{eV}$. For ${ }^{222} \mathrm{Rn}$ also the decays of progenies can be detected. Three $\alpha$-emitting nuclides are present in the decay chain before the long-lived ${ }^{210} \mathrm{~Pb}$ is reached. For a $50 \mathrm{keV}$ threshold the total counting efficiency was measured to be $(148 \pm 2) \%$ for the whole chain (Rau, 1999).

The minimum detectable activities can be estimated from the measured efficiencies and from the blank contribution of the line for gas purification and counter filling. For ${ }^{222} \mathrm{Rn}$, which has the highest concentration in the atmosphere from the nuclides of interest (see Table 1), the blank contribution was measured to be $(25 \pm 13) \mu \mathrm{Bq}$. For ${ }^{85} \mathrm{Kr}$ that contribution is negligible compared to the background of the counter, because of its low concentration in the atmosphere (see Table 1), and because it is not produced inside the system. The minimum detectable activity for radon is about $50 \mu \mathrm{Bq}$. For ${ }^{85} \mathrm{Kr}$ it is about $100 \mu \mathrm{Bq}$. The disadvantages due to the higher background at low energies and due to the lower counting efficiency are 
partly balanced by the lower blank contribution of the gas purification line and the much longer half-life.

\section{Applications to the BorEXINO experiment}

The BOREXINO experiment aims to detect the solar ${ }^{7} \mathrm{Be}$ neutrinos in real-time via elastic neutrino-electron scattering in an organic liquid scintillator (BOREXINO collaboration, Alimonti et al., 2002). The expected count rate of about 34 neutrino-induced events per day sets stringent limits for radioactive impurities in the liquid scintillator. Highly efficient purification techniques for organic liquids have been developed to match these requirements. Gaseous impurities are removed from the scintillator by nitrogen sparging, and consequently the nitrogen itself must also be very pure. The requirements for ${ }^{39} \mathrm{Ar},{ }^{85} \mathrm{Kr}$ and ${ }^{222} \mathrm{Rn}$ are discussed in detail in (Heusser et al., 2000; Zuzel et al., 2004) and are summarized in Table 1.

The developed techniques were used in the BOREXINO solar neutrino experiment to measure ${ }^{222} \mathrm{Rn}$ and ${ }^{85} \mathrm{Kr}$ in nitrogen. As described in chapter 3 the minimum detectable activities lie in the range of $50 \mu \mathrm{Bq}$ and $100 \mu \mathrm{Bq}$, respectively. Consequently krypton and radon have to be concentrated from several hundred cubic meters of nitrogen in order to reach the required minimum detectable activity concentrations.

It has been shown that about $150 \mathrm{~g}$ of activated carbon are sufficient to concentrate radon from at least $500 \mathrm{~m}^{3}$ (STP) of nitrogen, when the adsorption column is operated at liquid nitrogen temperature (Heusser et al., 2000). Due to the very different adsorption parameters of both gases nitrogen does not disturb the adsorption of radon. It changes when the components of a gas mixture have similar thermodynamical properties. This is the case for argon and nitrogen which have almost the same adsorption parameters. Therefore the argon is in competition with the nitrogen for the available adsorption sites. In consequence nitrogen can not be purified from argon by adsorption.

The situation regarding the concentration of krypton from nitrogen lies between the two cases of radon and argon. Although krypton has a somewhat larger adsorption parameter than nitrogen, the difference is small. Therefore there is still a competition between nitrogen and krypton for available adsorption sites. Two cases have to be distinguished: When the nitrogen is liquefied inside the trap, the adsorption of krypton is strongly hindered, because the adsorber is completely covered with nitrogen. The situation changes in the case of pure gas adsorption, when there are more free sites available for krypton. Although the same effect can be observed for radon, its impact is stronger in the case of krypton due to the similarity of the adsorption parameters. We proved the difference of liquid and gas phase adsorption with the following experiment:

Two traps filled with activated carbon were both immersed in liquid nitrogen and were connected in series. Nitrogen with a known krypton volume concentration of $3 \cdot 10^{-11}$ entered the first trap in the liquid phase. It was evaporated and heated up to about $450 \mathrm{~K}$ before it entered the second trap. Due to the high flow rate, the cooling was not sufficient to liquefy the nitrogen again. The final temperature in the second trap was about $100 \mathrm{~K}$. Consequently, there was pure liquid phase adsorption in the first trap and pure gas phase adsorption in the second trap. The process was stopped when the krypton concentration in both traps was in equilibrium between the adsorbed and the mobile phase. After removing most of the nitrogen with a vacuum-pump and a getter-pump, the krypton in both traps was measured with the 
method described in this paper. The result showed that the adsorption from pure gas phase was about two orders of magnitude more efficient than the liquid phase adsorption.

Consequently, gas phase adsorption has to be applied to improve the minimum detectable activity concentration for ${ }^{85} \mathrm{Kr}$ in nitrogen substantially. Tests with a noble gas mass spectrometer are performed to determine how much activated carbon is required to concentrate the krypton from $750 \mathrm{~m}^{3}$ of nitrogen (STP). Preliminary results show that a kilogram of activated carbon with an optimized pore-size distribution might be sufficient.

\section{Summary}

A highly sensitive technique was developed in the framework of the BOREXINO experiment to count the decay of nuclides of radioactive noble gases heavier than neon. It allows the detection of ${ }^{222} \mathrm{Rn}$ and ${ }^{85} \mathrm{Kr}$ at a minimum detectable activity of $50 \mu \mathrm{Bq}$ and $100 \mu \mathrm{Bq}$, respectively. The technique can be extended to xenon and argon allowing further applications in the future. The low detection limit was reached by the combination of an appropriate chromatographic separation procedure and the use of low background proportional counters. The technique is applied to the BOREXINO experiment to control the purity of nitrogen. For that reason adsorption columns have been developed that allow the concentration of noble gases from large amounts of nitrogen. Using these columns the minimum detectable activity concentrations can be improved sufficiently to check the stringent requirements in terms of ${ }^{85} \mathrm{Kr}$ and ${ }^{222} \mathrm{Rn}$ purity for the nitrogen used in the BOREXINO experiment. 


\section{Figures}

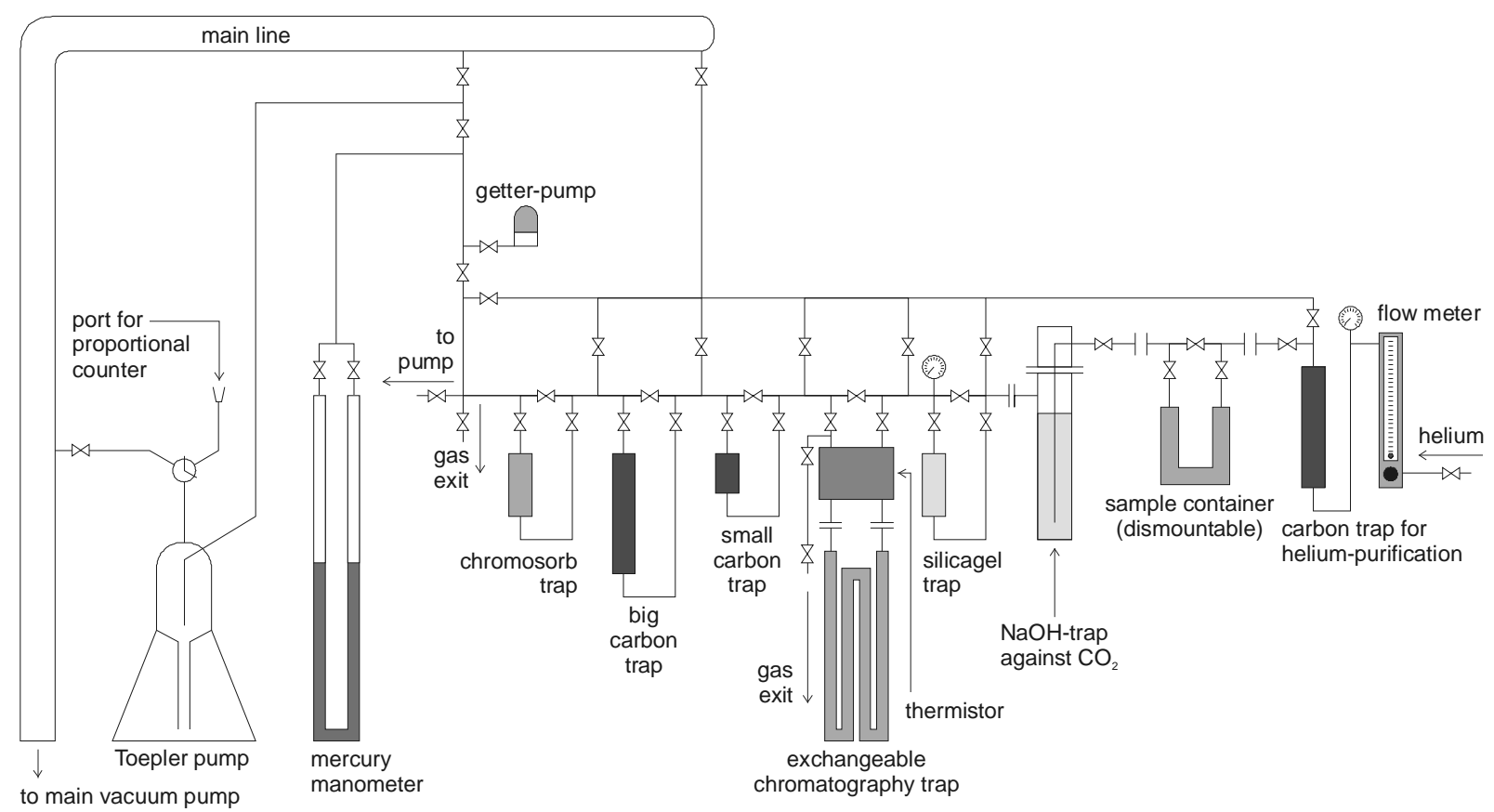

Figure 1: The glass vacuum line for the purification of noble gas samples and the filling of the proportional counters. 
Tables

\begin{tabular}{|c|c|c|c|}
\hline & ${ }^{39} \mathrm{Ar}$ & ${ }^{85} \mathrm{Kr}$ & ${ }^{222} \mathrm{Rn}$ \\
\hline \multirow{2}{*}{$\begin{array}{c}\text { Activity } \\
\text { concentration }\end{array}$} & $1.4 \mathrm{~Bq} / \mathrm{m}^{3}$ argon ${ }^{1)}$ & $1.4 \mathrm{MBq} / \mathrm{m}^{3} \mathrm{krypton}{ }^{2)}$ & - \\
\cline { 2 - 4 } & $13 \mathrm{mBq} / \mathrm{m}^{3}$ air & $1.4 \mathrm{~Bq} / \mathrm{m}^{3}$ air & $\begin{array}{c}\text { typically } 10-100 \mathrm{~Bq} / \mathrm{m}^{3}, \\
\text { sometimes higher }\end{array}$ \\
\hline Required $\mathrm{N}_{2}$ purity & $0.4 \mu \mathrm{Bq} / \mathrm{m}^{3}$ & $0.13 \mu \mathrm{Bq} / \mathrm{m}^{3}$ & $6 \mu \mathrm{Bq} / \mathrm{m}^{3}$ \\
\hline
\end{tabular}

${ }^{1)}$ Loosli, 1983; ${ }^{2)} \mathrm{BfS}, 2001$

Table 1: The most frequent radioactive noble gas nuclides in the atmosphere and the purity requirements for nitrogen to be used in the BOREXINO experiment (all gas volumes are given at STP). 


\section{References}

Bundesamt für Strahlenschutz (BfS), 2001. Künstliche Umweltradioaktivität. Jahresbericht, 63.

BOREXINO collaboration, Alimonti, G. et al., 2002. Science and technology of BoREXINO: a real-time detector for low energy solar neutrinos. Astroparticle Physics 16, 205-234.

Heusser, G. et al., 2000. ${ }^{222} \mathrm{Rn}$ detection at the $\mu \mathrm{Bq} / \mathrm{m}^{3}$ range in nitrogen gas and a new $\mathrm{Rn}$ purification technique for liquid nitrogen. Applied Radiation and Isotopes 52, 691-695.

Loosli, H.H., 1983. A dating method with ${ }^{39}$ Ar. Earth and Planetary Science Letters 63, 5162.

Maurer, S. et al., 2001. Henry coefficients of adsorption predicted from solid Hamaker constants. Chemical Engineering Science 56, 3443-3453.

Rau, W., 1999. Low-Level-Radonmessungen für das Sonnenneutrinoexperiment BOREXINO. $\mathrm{Ph} . \mathrm{D}$. thesis, Heidelberg university.

Rau, W. and Heusser, G., 2000. ${ }^{222}$ Rn emanation measurements at extremely low activities. Applied Radiation and Isotopes 53, 371-375.

Richter, S., 1988. Verfahren zur Herstellung von kleinen $\mathrm{GeH}_{4}-$ Mengen für das GalliumSonnen-Neutrinoexperiment. Diploma Thesis, Heidelberg university.

Schanda, U. and Urban, A., 1996. Energy and active volume calibration of Xe-filled proportional counters in the 1 to $10 \mathrm{keV}$ range. Nuclear Instruments and Methods A 381, 79-85.

Shriver, D.F., 1969. The Manipulation of Air-sensitive Compounds. McGraw-Hill, New York.

Simgen, H. et al., 2003a. A new system for the ${ }^{222} \mathrm{Rn}$ and ${ }^{226} \mathrm{Ra}$ assay of water and results in the BOREXINO project. Nuclear Instruments and Methods A 497, 407-413.

Simgen, H., 2003b. Hochempfindlicher Nachweis radioaktiver Edelgasnuklide und natürlicher Radionuklide aus der Uran-Zerfallsreihe. Ph.D. thesis, Heidelberg university.

Wink, R. et al., 1993. The miniaturized proportional counter HD-2(Fe)/(Si) for the GALLEX solar neutrino experiment. Nuclear Instruments and Methods A 329, 541-550.

Zuzel, G. et al., 2004. Ar and Kr concentrations in nitrogen as a measure of the ${ }^{39} \mathrm{Ar}$ and ${ }^{85} \mathrm{Kr}$ activity in connection with the solar neutrino experiment BOREXINO. Appl. Rad. Isot., this issue. 